\title{
Differential feature analysis in the recognition memory of reflective and impulsive children*
}

\author{
RICHARD R. KILBURG \\ W'estern Psychiatric Institute and Clinic, Pittsburgh, Pennsylvania 15213 \\ and \\ ALEXANDER W. SIEGEL \\ University of Pittsburgh, Pittsburgh, Pennsylvania 15260
}

\begin{abstract}
Seventy-two children. 18 reflective, and 18 impulsive first and fifth graders were tested in a forced-choice recognition memory task. Experimental conditions systematically varied the possibility that correct responses could be made on the basis of verbal labels, visual feature analyses, or both. Although reflective children made more correct recognition choices than did impulsive children under all experimental conditions, this difference was, as predicted, significant only in a condition in which the sole basis for correct response depended on a purely visual feature analysis. Order of condition difficulty indicated that visual feature analysis independent of verbal processes was responsible for successful recognition performance in these Ss. Results were discussed with reference to the feature-testing model for recognition proposed by Selfridge and Neisser.
\end{abstract}

Kagan and Kogan's (1970) review of the literature on individual variation in cognitive processes indicates that the dimension of reflection-impulsivity is a reliable and useful dimension along which to conceptualize cognitive style. The matching familiar figures test (MFF) is a reliable means of evaluating a child's relative position on the dimension of cognitive style which Kagan, Rosman, Day, Albert, and Phillips (1964) have called reflection-impulsivity. In the MFF, $\mathrm{S}$ is shown a standard stimulus and asked to choose the one of several strikingly similar variants that exactly matches the standard. Children who respond slowly and make relatively few errors are classified as "reflective," while children who respond quickly and make many errors are classified as "impulsive." A variety of studies have shown consistent performance differences on a variety of measures: Reflective children make fewer reading errors (Kagan, 1965), fewer errors of commission on a serial learning task (Kagan, 1966), fewer errors on inductive reasoning tasks (Kagan, Pearson, \& Welch, 1966), and tend to focus more on a hypothesis testing task (Nuessle, 1972). In general, these studies have implied that, at a given age level, the more reflective the

\footnotetext{
*This research is derived from a project of the Learning Research and Development Center, University of Pittsburgh, supported in part as a research and development center by funds from the United States Office of Education, Department of Health, Education, and Welfare. The opinions expressed in this publication do not necessarily reflect the position or policy of the Office of Education, and no official endorsement by the Office of Education should be inferred. The authors would like to acknowledge the cooperation of Sister Collette, Superintendent of the Pittsburgh Parochial Schools, for supplying $S$ s and of Kathleen Kirasic for running the matching familiar figures test.

†Requests for reprints should be sent to Alexander W. Siegel, Department of Psychology. University of Pittsburgh, Pittsburgh. Pennsylvania 15260.
}

$S$ is, the more differentiated and adequate the scanning strategy he employs during the interval between stimulus presentation and his initial response in a task involving a variety of response alternatives.

In addition to the research extending the validity of the concept of reflection-impulsivity (R-I), several recent studies have experimentally investigated relationships between R-I and visual scanning strategies on the MFF. Siegelman (1969) found that impulsive fourth graders ignored $2 \frac{1}{2}$ times as many variants per item as did reflective fourth graders, while reflective fourth graders distributed their attention more evenly across the standard and the variants. Drake (1970) recorded eye movements while $\mathrm{S}$ was performing the MFF and found that impulsive third graders made fewer standard-variant comparisons on the MFF than did reflective third graders. Zelniker, Jeffrey, Ault, and Parsons (1971) recorded eye fixations on the MFF and proposed that impulsive children have less adequate strategies for searching the stimulus complex. Odom, McIntyre, and Neale (1971) compared the perceptual learning of reflective and impulsive kindergarten children; the performance of the reflective $S$ s indicated that they perceived and evaluated information based on the feature differences of stimulus arrays; the information processed by the impulsive Ss could not be identified.

Although these studies suggest that reflective and impulsive children differ in their perceptual approach to the task and Drake and Odom et al suggest that reflective and impulsive children perform different kinds of feature analyses of stimulus arrays, these suggestions remain speculations as to the cognitive basis for these performance differences and clearly are not based on any coherent theory. One theoretical source for explaining the hypothesized differences in feature analysis during the interval between stimulus 
presentation and response is the decision model developed by Selfridge (1959, "Pandemonium") and Neisser (Neisser, 1967; Selfridge \& Neisser, 1961). Since one criterion in deciding whether or not an individual is reflective or impulsive is response latency and since the Selfridge-Neisser model can account for most of the choice reaction-time research data (Smith, 1968), the model might be quite useful in accounting for some of the performance differences between reflective and impulsive children.

The Selfridge-Neisser model is hierarchical and is based mainly on a program for letter recognition that emphasizes feature testing. The model assumes that there are several levels of mechanisms operating to decide the fate of incoming information: Level 1 mechanisms are stimulus samplers that get basic information into the system; Level 2 mechanisms are a number of stimulus analyzers, each of which determines whether or not the stimulus has (i.e., the extent to which the stimulus is characterized by) certain features. Results of these feature tests are conveyed to the next level, a set of "subroutines" which perform operations on the results of the feature tests. At the highest level, the sums from these subroutines are compared and the item associated with the largest sum is selected as the best "guess" as to the identity of the stimulus. Another feature of the model is that tests at the same level may be carried out simultaneously. Thus, the time required to "categorize" a stimulus at any given level does not depend on the number of feature tests performed, and a longer response latency might well reflect a difference in the depth of the feature analysis performed or the time required to make a decision.

On the basis of this model, it can be hypothesized that the differences between reflective and impulsive children are not as likely due to the reflective S's performing more adequate stimulus sampling at Level 1 as due to their performing a more detailed and thorough feature analysis at Level 2 and/or having a more rigorous decision criterion. Impulsive performance might entail fewer stimulus samples drawn by Level 1 mechanisms but would more likely result in fewer features of the stimulus being tested and, consequently, the impulsive S's decision would be made on the basis of a poorly defined alternative. The findings of Siegelman (1969), Drake (1970), and Odom et al (1971) could be interpreted as indicating that reflective Ss are drawing more stimulus samples to form the features for the alternatives in the decision-making process but are also performing a more detailed feature analysis. Prior to further speculation concerning the specific level of the model at which R-I differences would be determined, it is first necessary to demonstrate that reflective children perform differently than impulsive children on a task (other than the MFF) requiring visual feature analysis.

The specific purpose of this study was to demonstrate that reflective and impulsive first and fifth graders would differ significantly in their tendency to perform a detailed visual feature analysis, as measured by their performance on a forced-choice recognition memory task in which feature differences were manipulated systematically.

\section{METHOD}

\section{Subjects}

One hundred seventy white middle-class boys, 74 first graders and 96 fifth graders, attending Pittsburgh parochial schools participated in the research. The final sample consisted of 18 reflective (mean $\mathrm{CA}=7$ years 2 months, range $=6.7$ to $8-4$ ) and 18 impulsive (mean $\mathrm{CA}=7-2$, range $=6-7$ to $7-11$ ) first-grade boys and 18 reflective (mean CA $=11-3$, range $=10-11$ to $11-9$ ) and 18 impulsive (mean $\mathrm{CA}=11-1$, range $=10-8$ to $11-8$ ) fifth-grade boys.

\section{Materials}

The matching familiar figures test (Kagan et al, 1964) was used to determine reflective-impulsive classification. The MFF is a match to sample task in which $S$ is shown a standard stimulus and told to choose the one of six strikingly similar variants that exactly matches the standard. Two practice and 10 test items were given.

Stimuli for the recognition memory task consisted of a presentation deck and a test deck. The initial deck consisted of $803 \times 5$ in. laminated cards, on each of which was a black line drawing of a common object or animal. The test deck consisted of $805 \times 8$ in. laminated cards, on each of which were two black line drawings.

\section{Apparatus}

The apparatus consisted of a test stand on which each of the test cards was placed. At the bottom of the stand was a photocell-controlled microswitch, wired to a Hunter timer (facing E) that started each time a new card was placed on the stand. In front of the stand and below the test card locus were two response buttons. When either button was depressed, the timer automatically stopped. E manually recorded response latency from the time between stimulus presentation and selection.

\section{Procedure}

All 170 children were individually administered the MFF during a first session lasting approximately $20 \mathrm{~min}$. The essential instructions to the child were that he was always to point to the variant on the lower page that was just exactly like the standard (on the upper page). For each of the 10 test items, E recorded the number of errors the child made on each item (a maximum of three errors per item was allowed) and the response latency for each item (time from stimulus presentation to first response, whether correct or not). For each age level, children whose mean response latency was above the median and whose total number of errors was below the median were classified as reflective; children whose mean response latency was below the median and whose total number of errors was above the median were classified as impulsive. Of the 74 first graders, 24 were classified as reflective and 22 as impulsive; of the 96 fif th graders, 35 were classified as reflective and 30 as impulsive. Due to time constraints, it was not possible to test all of the reflective and impulsive children and, thus, 18 children were randomly selected from each Grade X R-I subgroup. Intelligence scores were available only for the fifth graders. The mean Otis-Lennon IQ of the 18 reflective $S \mathrm{~S}(\overline{\mathrm{X}}=105.7)$ was not significantly different from that of the 18 impulsive Ss $[\bar{X}=109.7 ; t(34)<1$. $\mathrm{p}>.10]$. 
Fig. 1.
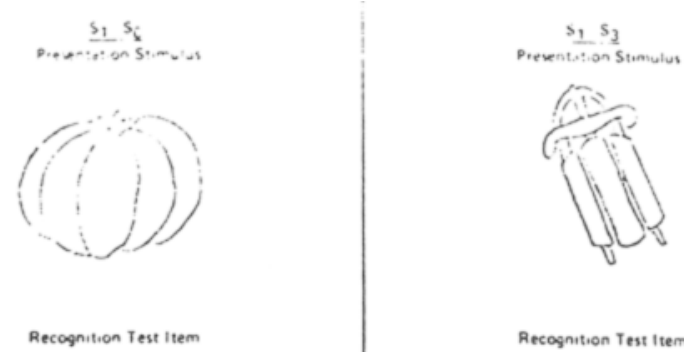

Recognition Test Item
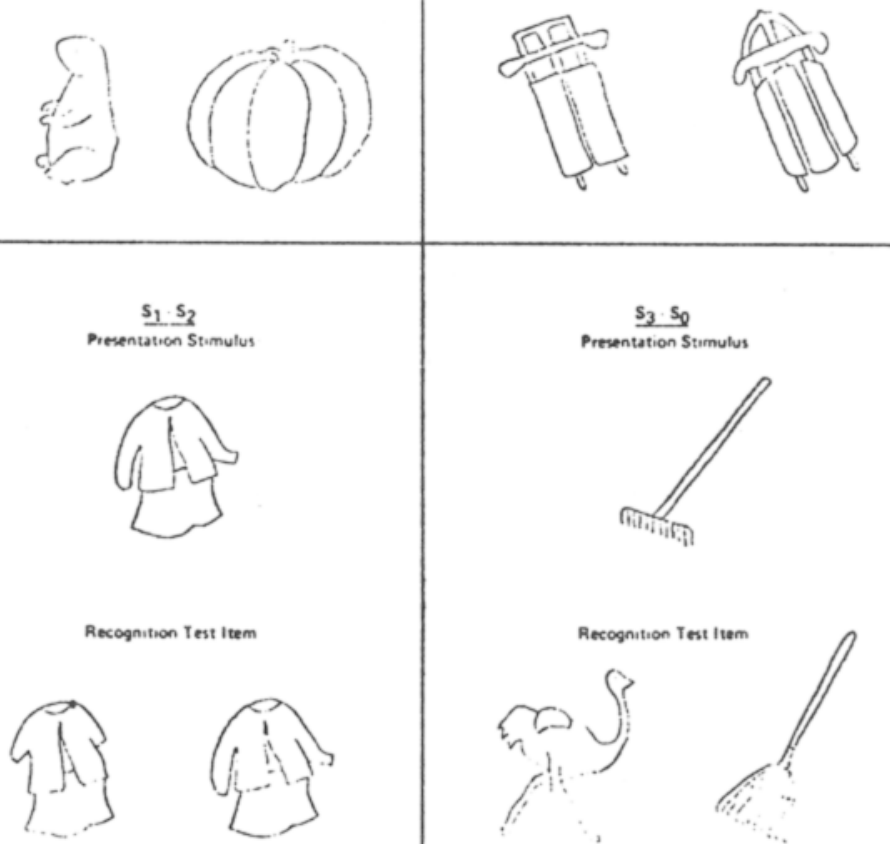

In a second session held approximately 2 weeks later, these 72 children were individually administered the test of recognition memory by a different $E$ (RRK). Each $S$ was seated, handed the presentation deck of 80 cards, and given the following instructions: "Here is a deck of cards with drawings on them of animals and things that you have seen before. I want you to look carefully at each of the cards and go through the deck as quickly as you can. When you finish looking at each card, turn it over and put it in a pile over there." E recorded the total time $S$ took to go through the presentation deck.

Following the initial sorting task, each $\mathrm{S}$ was told: "Now I'm going to show you some more cards. Each one has two drawings on it. I'll put each card on the stand in front of you. I want you to look at both drawings and push the button underneath the one you saw before in the first part of the game. Push the button as quickly as you can af ter you make your choice. If you are not sure which one it is. I want you to guess anyway." Each $S$ was then shown all 80 test cards. one at a time. For each test card, $E$ recorded whether the response was correct or incorrect and the latency of that response.

\section{Experimental Conditions}

The recognition test deck consisted of 80 cards. 20 from each of four experimental conditions. Within each deck of 20. the correct figure was in the left position on 10 of the cards and in the right position on the other 10 . All children saw the 80 test stimuli in the same completely randomized order. Examples of presentation and recognition test items for ath experimental condition are presented in lig. 1.

\section{(1) Condition $\left(S_{1}-S_{0}\right)$}

Twenty stimuli were chosen randomly from the 80 original stimuli $\left(S_{1}\right)$ and each was paired with a completely new animal or object on the test card (see Fig. 1a). This condition should be the easiest (i.e., should produce the most correct recognition responses), since a correct response can be made either on a global feature analysis and/or on the basis of the name of the $\left(S_{1}\right)$ stimulus. Since Rosinski (1970) has found that the recognition performance of fifth graders was superior to that of first graders under a similar stimulus condition, it was expected that fifth graders would make more correct recognition responses on these stimuli than would first graders. Since a detailed visual feature analysis was not required in order to make a correct response and since it was hypothesized that reflective and impulsive Ss differ primarily in their tendency to perform detailed feature analyses. the performance of reflective $S s$ was not expected to differ from that of impulsive Ss.

\section{(2) Condition $\left(S_{1}-S_{2}\right)$}

Twenty different stimuli from the original $80\left(S_{1}\right)$ stimuli were each paired with another stimulus having the same name. draw $n$ in the same style. and differing from $S_{1}$ in only one minor feature $\left(S_{2}\right)$ (see Fig. lb). This condition should be the most difficult. since choosing the correct stimulus requires a rather complete feature analysis of the original $\left(S_{1}\right)$ stimulus during initial presentation. and the correct choice camnot be made on the basis of the name of the stimulus. If these stimuli are not so difficult as to produc shance performance. then retlective so 
should make more correct recognition responses than impulsive Ss.

\section{(3) Condition $\left(S_{1}-S_{3}\right)$}

Twenty different stimuli from the original $80\left(S_{1}\right)$ were paired with another stimulus having the same name but drawn in a very different style and differing from $S_{1}$ in several different details $\left(S_{3}\right)$ (see Fig. 1c). This condition should be of intermediate difficulty: The $\left(S_{1}-S_{3}\right)$ stimuli should be easier to discriminate than the $\left(S_{1}-S_{2}\right)$ stimuli, since there are more feature differences, but should be harder to discriminate than the $\left(S_{1}-S_{0}\right)$ stimuli, since the correct choice still cannot be made on the basis of the name of the stimulus. As in $\left(S_{1}-S_{2}\right)$. reflective $S s$ should make more correct recognition responses than impulsive $S$ s.

\section{(4) Condition $\left(S_{3}-S_{0}\right)$}

The remaining 20 stimuli from the presentation deck were redrawn in a similar fashion as the $S_{3}$ stimuli in $\left(S_{1}-S_{3}\right)$ and were each paired with a completely new animal or object on the test card (see Fig. 1d). If, as Rosinski (1970) and Neisser(1967) have argued, recognition memory is primarily a visual process, then performance in this condition should be relatively poor: $A$ correct recognition response can be made only on the basis of the name of the stimulus (or a global template), not on the basis of specific detailed visual features. Thus, the performance of reflective and impulsive $S s$ was not expected to differ in this condition.

It should be noted that differences in the performance of reflective and impulsive $S$ s were predicted only in the two conditions in which a correct recognition response could be made only on the basis of different visual features $\left(S_{1}-S_{2}\right)$ and $\left(S_{1}-S_{3}\right)$ and not in conditions in which the correct response could be made on the basis of the name of the stimulus.

If, as Kagan (1966) has argued, a child's tendency to long or short response times (conceptual tempo) is consistent across a variety of perceptual (visual) tasks, then the response latencies of reflective Ss should be significantly greater than those of impulsive Ss. As has been found in a variety of tasks, response speed decreases with developmental level. Thus, it was expected that the latencies of first graders would be greater than those of the fifth graders. No differential predictions were made concerning specific differences in response latencies among the four experimental conditions.

\section{Experimental Design}

The experimental design was a 2 (grade) by 2 (reflection-impulsivity) by 18 (Ss) by 4 (stimulus conditions) mixed factorial design with repeated measures on the last factor.

\section{RESULTS}

\section{MFF Data}

For the entire sample of first graders, the median latency for MFF items was $9.4 \mathrm{sec}$ and the median number of errors was 16 . The mean MFF latency and errors of the 18 reflective Ss were $15.17 \mathrm{sec}$ and 11.22 errors; the mean MFF latency and errors of the 18 impulsive Ss were $5.72 \mathrm{sec}$ and 19.33 errors.

For the entire fifth-grade sample, the median latency for MFF items was $8.3 \mathrm{sec}$ and the median number of errors was 9. The mean MFF latency and errors of the 18 reflective Ss were $13.52 \mathrm{sec}$ and 5.28 errors: the mean
MFF latency and errors of the 18 impulsive Ss were $5.48 \mathrm{sec}$ and 14.33 errors.

Correlations between MFF errors and mean MFF latency were highly significant for both first $[r(72)=-.54, \quad p<.001]$ and fifth graders $[\mathrm{r}(94)=-.50, \mathrm{p}<.001]$. The magnitude of this correlation very closely approximates that found by Kagan et al (1964) and Nuessle (1972) for Ss of similar age levels.

For the sample as a whole, MFF errors were essentially unrelated to any of the recognition memory measures (i.e., sorting time, number of correct responses in each condition, and latency in each condition) $[\mathrm{r}(70) \leqslant .23, \mathrm{p}>.10]$. MFF latency, on the other hand, was significantly related to the other latency measures (sorting time and latency in each condition) $[r(70) \geqslant .26, p<.05]$ but was unrelated to the number of correct choices in any condition $[\mathrm{r}(70) \leqslant .13$, $p>.10]$.

\section{Recognition Memory Task}

\section{(1) Sorting Time on Presentation Deck}

A 2 (grade) by 2 (R-I) analysis of variance performed on the number of seconds each $S$ required to complete the initial presentation task yielded significant main effects of grade and R.I $[F(1,68) \geqslant 4.41, \mathrm{p}<.05]$ : First graders took longer to go through the presentation deck $(157.8 \mathrm{sec})$ than did the fifth graders $(137.7 \mathrm{sec})$, and reflective Ss took longer (158.5) than did impulsive Ss (136.7). An alternative way of describing these results is that, on the average, reflectives and first graders spent approximately $.25 \mathrm{sec}$ more looking at (or manipulating) each presentation card than did impulsive and fifth-grade Ss.

\section{(2) Recognition Task Performance: Correct Responses}

The means and standard deviations of the number of correct responses made under each of the four experimental conditions by each grade and R-I group are presented in Table 1. Ss' performance under each of the four conditions was ranked in the same order for the entire sample for both grade and R-I levels (from best to worst $):\left(S_{1}-S_{0}\right)>\left(S_{1}-S_{3}\right)>\left(S_{3}-S_{0}\right)>\left(S_{1}-S_{2}\right)$. Mean performance of the entire sample in Conditions $\left(S_{1}-S_{0}\right),\left(S_{1}-S_{3}\right)$, and $\left(S_{3}-S_{0}\right)$ was significantly greater than chance $(z \geqslant 2.24, p<.05)$, whereas performance in Condition $\left(S_{1}-S_{2}\right)$ was not $(z<1)$. This ordering of performance is essentially in line with predictions and is consistent across grade level and cognitive style. Since differences among conditions had been predicted, but the particular pairwise comparisons that would be significantly different were not specified, the conservative technique of Scheffé confidence intervals was used to test for specific pairwise 
Table 1

Means and Standard Deviations for Each Grade and R-I Level on the Total Number of Correct Responses for Each Experimental Condition

\begin{tabular}{|c|c|c|c|c|c|c|c|c|c|}
\hline \multirow[b]{3}{*}{ Group } & \multirow[b]{3}{*}{$\mathrm{N}$} & \multicolumn{8}{|c|}{ Experimental Conditions } \\
\hline & & \multicolumn{2}{|c|}{$S_{1}-S_{0}$} & \multicolumn{2}{|c|}{$\mathrm{S}_{1}-\mathrm{S}_{3}$} & \multicolumn{2}{|c|}{$S_{3}-S_{0}$} & \multicolumn{2}{|c|}{$S_{1}-S_{2}$} \\
\hline & & Mean & SD & Mean & SD & Mean & SD & Mean & $\mathrm{SD}$ \\
\hline First Graders & 36 & 14.00 & 2.31 & 13.48 & 2.50 & 10.97 & 2.75 & 9.86 & 2.62 \\
\hline Fifth Graders & 36 & 15.19 & 2.18 & 14.25 & 2.33 & 11.11 & 1.92 & 10.36 & 1.58 \\
\hline Reflective Ss & 36 & 14.94 & 2.22 & 14.42 & 2.37 & 11.17 & 2.27 & 10.06 & 2.11 \\
\hline Impulsive Ss & 36 & 14.25 & 2.37 & 13.31 & 2.40 & 10.92 & 2.47 & 10.17 & 2.25 \\
\hline Total Sample & 72 & 14.60 & 2.31 & 13.86 & 2.43 & 11.04 & 2.36 & 10.11 & 2.16 \\
\hline
\end{tabular}

comparisons. Scheffé $(.01)$ confidence intervals [pooled within mean square error, $\mathrm{df}(204)=3.88$, critical value $=$ $1.12]$ indicated that the performance of Ss on Condition $\left(\mathrm{S}_{1}-\mathrm{S}_{0}\right)$ (mean $=73 \%$ correct) did not differ from that on Condition $\left(S_{1}-S_{3}\right)$ (mean $=69 \%$ correct), nor did performance on $\left(S_{3}-S_{0}\right)$ (mean $=55 \%$ correct) differ from that on $\left(S_{1}-S_{2}\right)$ (mean $=51 \%$ correct $)$. However, as predicted, performance on both Conditions $\left(S_{1}-S_{0}\right)$ and $\left(S_{1}-S_{3}\right)$ was significantly greater than that on both Conditions $\left(S_{1}-S_{2}\right)$ and $\left(S_{3}-S_{0}\right)$. That performance on $\left(S_{1}-S_{3}\right)$ was equivalent to that on $\left(S_{1}-S_{0}\right)$ and performance in both was greater than that on $\left(S_{3}-S_{0}\right)$ indicates that the role of verbal labels appears to have little or no direct effect on recognition performance.

Since specific predictions had been made regarding grade and R-I differences in the four conditions, these hypotheses were evaluated using the method of planned comparisons specified by Hays (1963, Chapter 14). As predicted and in line with Rosinski's (1970) results, fifth graders made significantly more correct responses than did first graders on Condition $\left(\mathrm{S}_{1}-\mathrm{S}_{0}\right)$ [SE of mean $=$ $.465, \mathrm{t}(68)=2.56, \mathrm{p}<.01$, one-tailed]. In line with the prediction that reflective and impulsive Ss would differ only on the conditions in which correct responses could be made only on the basis of visual feature differences, the performance of reflective Ss was not significantly better than that of impulsive Ss on this condition [SE of mean $=.465, \mathrm{t}(68)=1.48, \mathrm{p}>.10 \mathrm{~J}$.

Condition $\left(S_{1}-S_{2}\right)$ was the most difficult condition and the only one in which performance did not differ from chance for either grade or cognitive style level. This condition appeared to require a feature analysis too detailed for any of the children and, thus, a "floor effect" was obtained: The performance of reflective and impulsive Ss did not differ $(t<1)$.

As predicted, reflective Ss made significantly more correct responses than did impulsive $S$ s on Condition $\left(\mathrm{S}_{1}-\mathrm{S}_{3}\right)$ [SE of mean $=.465, \mathrm{t}(68)=2.39, \mathrm{p}<.01$. one-tailed]. This crucial finding confirmed the prediction that, in a condition in which the only basis for correct recognition was visual feature differences. reflective Ss would perform better than impulsive Ss. Since the performance of fifth graders was not significantly better than that of first graders on this condition [SE of mean $=.465, \mathrm{t}(68)=1.61, \mathrm{p}>.10$ ], it appeared that the capacity for purely visual feature analysis does not increase with developmental level.

Finally, the performance of reflective Ss did not differ significantly from that of impulsive $S s$ on Condition $\left(S_{3}-S_{0}\right)(S E$ of mean $=.465, t<1)$. This also confirms the prediction that reflective and impulsive Ss would not differ on a condition in which the name of the stimulus was the only basis for correct recognition.

In summary, reflective Ss performed significantly better than impulsive Ss only in Condition $\left(S_{1}-S_{3}\right)$. Correct recognition in this condition could be made only on the basis of visual features, correct recognition in $\left(S_{1}-S_{0}\right)$ could be made on the basis of either visual features or the name of the stimulus, and correct recognition in $\left(S_{3}-S_{0}\right)$ could be made only on the basis of the name of the stimulus. That performance on $\left(S_{1}-S_{3}\right)$ was equivalent to that on $\left(S_{1}-S_{0}\right)$ and performance on both of these was greater than on $\left(S_{3}-S_{0}\right)$ provides strong inferential evidence that recognition memory is primarily a process of visual feature analysis; the role of "verbal mediation" seems to be minimal. Although fifth graders made more correct responses than first graders in all four conditions, this difference was significant only in $\left(S_{1}-S_{0}\right)$-the "standard" recognition memory task. Thus, capacity to perform visual feature analyses seems not to increase between first and fifth grade.

\section{(3) Recognition Task Performance: Latencies}

Since overall R-I, grade, and condition effects had been predicted (rather than specific comparisons), a 2 (grade) by 2 (R-I) by 18 (Ss) by 4 (conditions) analysis of variance was performed on each S's mean latency' for each of the four experimental conditions. Only the main effect of experimental conditions was significant $[F(3,204)=35.38, p<.001]$. The mean latencies in the four experimental conditions were consistently ordered (from fastest to slowest): $\left(S_{1}-S_{0}\right),\left(S_{1}-S_{3}\right)$. $\left(S_{3}-S_{0}\right),\left(S_{1}-S_{2}\right)$, thus mirroring the ordering of correct responses. The easier a given condition (i.e.. the more correct responses made). the shorter the response latency for that condition. Scheffé (.01) contidence interials fpooled with mean square error. 
$\operatorname{df}(204)=0.72$, critical value $=0.71]$ indicated that the mean latency in Condition $\left(S_{1}-S_{0}\right)(3.03 \mathrm{sec})$ did not differ from that in Condition $\left(S_{1}-S_{3}\right)(3.17)$, nor did the latency in Condition $\left(S_{3}-S_{0}\right)$ (3.89) differ from that in $\left(S_{1}-S_{2}\right)$. However, the mean latencies in both Conditions $\left(S_{1}-S_{0}\right)$ and $\left(S_{1}-S_{3}\right)$ were significantly shorter than those in both Conditions $\left(S_{3}-S_{0}\right)$ and $\left(S_{1}-S_{2}\right)$. Although the overall mean latency of the reflective was greater than that of the impulsive Ss (3.83 vs $3.36 \mathrm{sec}$ ) and that of the first graders was greater than that of the fifth graders $(3.85 \mathrm{vs} 3.34 \mathrm{sec})$, contrary to prediction, neither the main effect of R-I nor grade was significant $[F(1,68) \leqslant 1.73, p>.10]$.

\section{DISCUSSION}

In general, the results of the present study indicated that there are performance differences between reflective and impulsive children on a task requiring visual feature analyses and, thus, are congruent with other earlier findings of Drake (1970), Siegelman (1969) and Zelniker et al (1972). However, it also seems clear that the data argue against the hypothesis that the nature of the search process is different for reflective and impulsive Ss. That is, the differential performance of reflective and impulsive $S s$ is due to a quantitative difference in the thoroughness of the process of feature analysis rather than to a qualitative or process difference. That the relative ordering of performance in the four conditions was similar for both reflective and impulsive Ss and that in all conditions the performance of reflective Ss was greater than that of the impulsive Ss [but significant only in Condition $\left(S_{1}-S_{3}\right)$ ] seems to substantiate this reasoning. It would appear that, in a task in which the only basis for correct responding is that of visual features, reflective Ss tend to perform a more detailed feature analysis. A recent study by Ault, Crawford, and Jeffrey (1972) has also found that reflective and impulsive Ss used the same basic strategy of making comparisons between the standard and one variant or between two variants on the MFF, but reflective $S s$ made a greater proportion of these comparisons.

The results obtained with Condition $\left(S_{1}-S_{3}\right)$ in particular are interpretable within the framework of the Selfridge-Neisser feature testing model of recognition: Both reflective and impulsive Ss tend to utilize the same feature analysis process, but reflective $S$ s tend to do a more thorough job. Although the present experimental design is insufficient to specify the level in the model at which these performance differences occur (more adequate stimulus sampling at Level 1 and/or a more detailed and thorough feature analysis at Level 2 and/or a more stringent decision criterion at a higher level), the results are sufficiently promising to warrant further systematic recognition memory research within the framework of a hierarchically organized feature testing model of recognition.
Another relatively clear implication of the present study can be drawn from the relative ordering of condition difficulty. The experimental conditions were so designed that, if recognition memory were a verbally mediated process, then Conditions $\left(S_{1}-S_{0}\right)$ and $\left(S_{3}-S_{0}\right)$ would have produced approximately the same level of performance. In Condition $\left(S_{3}-S_{0}\right)$, a correct response could be made only on the basis of the name of the stimulus. In addition, performance on Condition $\left(S_{1}-S_{3}\right)$ should have been relatively poor, as a correct response could not be made on the basis of the name of the stimulus. [The same argument should apply to $\left(S_{1}-S_{2}\right)$, but clearly this condition was just too difficult for the children tested.] These results were not obtained. Since performance on Condition $\left(S_{1}-S_{3}\right)$ was equivalent to that on $\left(S_{1}-S_{0}\right)$ and since performance on both was better than on $\left(S_{3}-S_{0}\right)$, it seems relatively clear that verbal mediation has relatively little influence on recognition memory. Rather, recognition memory seems to be largely dependent on a purely visual process of feature analysis. Although it seems intuitively likely that verbal labels should enhance recognition performance, it is also highly likely that their role is an indirect one, in the sense that in order to label a stimulus, feature analysis must first be performed and, thus, a label would serve to increase the probability of a thorough feature analysis.

A third implication of the present study which supports Kagan's (1965a) argument is that, since MFF items cannot be correctly solved on the basis of the name of the stimulus, the tendency to perform detailed visual feature analysis is perhaps the most significant component of the cognitive-perceptual basis underlying the dimension of reflection-impulsivity.

\section{REFERENCES}

Ault, R. L., Crawford, D. E., \& Jeffrey, W. E. Visual scanning strategies of reflective, impulsive, fast-accurate, and slow-inaccurate children on the Matching Familiar Figures Test. Child Development, 1972, 43, 1412-1417.

Drake, D. M. Perceptual correlates of impulsive and reflective behavior. Developmental Psychology, 1970, 2, 202-214.

Hays, W. L. Statistics for psychologists. New York: Holt, Rinehart, \& Winston, 1963. Chapter 14.

Kagan, J. Reflection-impulsivity and reading ability in primary grade children. Child Development, 1965, 36, 609-628.

Kagan, J. Reflection-impulsivity: The generality and dynamics of conceptual tempo. Journal of Abnormal Psychology, 1966, $71,17-24$.

Kagan, J., \& Kogan, N. Individual variation in cognitive processes. In P. H. Mussen (Ed.), Carmichael's manual of child psychology. Vol. 1. New York: Wiley, 1970. Pp. 1366-1378.

Kagan, J., Pearson, L., \& Welch, L. Conceptual impulsivity and inductive reasoning. Child Development, 1966, 37, 583-594.

Kagan, J., Rosman, B. L., Day, D., Albert, J., \& Phillips, W. Information processing in the child: Significance of analy tic and reflective attitudes. Psychological Monographs, 1964. 78(1. Whole No. 578). 
Neisser. L'. Cognitive psychology. New York: Appleton-Century-Crofts, 1967.

Nuessle. W. Reflectivity as an influence on focusing behavior of children. Journal of Experimental Child Psychology, 1972, 14. 265-276

Odom, R. D.. MeIntyre, C. W., \& Neale, G. S. The influence of cognitive style on perceptual learning. Child Development, $1971,42.883-892$.

Rosinski, R. R. Development of attention and recognition memory. Cinpublished doctoral dissertation, Cornell University. 1970.

Selfridge, O. G. Pandemonium: A paradigm for learning. In Mechanization of thought processes. London: Her Majesty's Stationery Office, 1959. Pp. $511-526$.

Selfridge, O. G., \& Neisser, U. Pattern recognition by machine.
Scientific American, 1960, 203, 60-68.

Siegelman, E. Refiective and impulsive observing behavior. Child Development, 1959, 40, 1213-1222.

Smith, E. E. Choice reaction time: An analysis of the major theoretical positions. Psychological Bulletin, 1968, 69, $77-110$.

Zelniker, T., Jeffrey, W. E., Ault, R., \& Parson, J. Analysis and modification of search strategies of impulsive and reflective children on the Matching Familiar Figures Test. Child Development, 1971, 43, 321-335.

(Received for publication February 16, 1973; revision received February 21,1973 ; accepted March 21, 1973.) 